\title{
Increased prevalence of differentiated thyroid carcinoma in patients with acromegaly
}

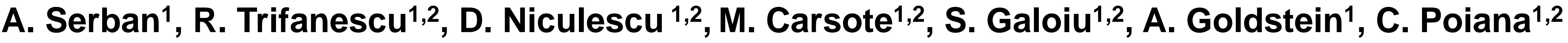 1 "C.I. Parhon" National Institute of Endocrinology, ${ }^{2}$ Dept. of Endocrinology, "Carol Davila" University of Medicine and Pharmacy, Bucharest, Romania}

\section{BACKGROUND}

Acromegaly, a rare chronic disease characterised by hypersecretion of growth hormone $(\mathrm{GH})$ and subsequently insulin growth factor 1 (IGF1), was associated with an increased prevalence of benign and malignant tumors $(1,2)$. Thyroid nodules were more frequently found in acromegalic patients than in general population with an OR $=3.6$ (3), and some studies suggested that the thyroid carcinoma is the most frequent type of cancer in the acromegalic patients $(4,5)$.

Aim: to assess the prevalence of thyroid nodules and thyroid carcinoma in patients with acromegaly.

\section{PATIENTS AND METHODS}

Patients: 40 acromegalic patients $\left(\begin{array}{lll}30 & F / 10 & M\end{array}\right)$ were retrospectively reviewed.

Methods: $\mathrm{GH}$ and IGF1 were measured by chemiluminescence (Liaison), TSH and FT4 by immunometric assays (Immulite).

Thyroid ultrasonography was performed in all cases, thyroid scintigraphy when indicated, and fine needle aspiration biopsy (FNAB) in suspicious nodules according to current guidelines.

\section{RESULTS}

\section{Patients' characteristics}

Gender

lodine deficient area

Age (years)

BMI (kg/m2)

Diabetes mellitus
$30 \mathrm{~F}: 10 \mathrm{M}$
$14(35 \%)$
$47 \pm 12.8$
$27.9 \pm 5.62$
$5(12.5 \%)$

last nadir GH /TTGO (ng/ml)

last IGF 1 (ULN * \%)

acromegaly duration (years)

controlled acromegaly
$4.2 \pm 13.9$

$146 \pm 90$

$7.6 \pm 9.5$

$10(25 \%)$

\section{Thyroid Disorders $=70 \%(28)$}

$\begin{array}{ll}\text { Diffuse goiter } & 8(20 \%) \\ \text { Nodular goiter } & 19(47.5 \%) \\ \quad \text { Simple nodular goiter } & 5(12.5 \%) \\ \text { Nontoxic multinodular goiter } & 10(25 \%) \\ \text { Toxic multinodular goiter } & 4(10 \%) \\ \text { Graves disease } & 1(2.5 \%) \\ \text { Thyroid carcinoma } & 3(7.5 \%)\end{array}$

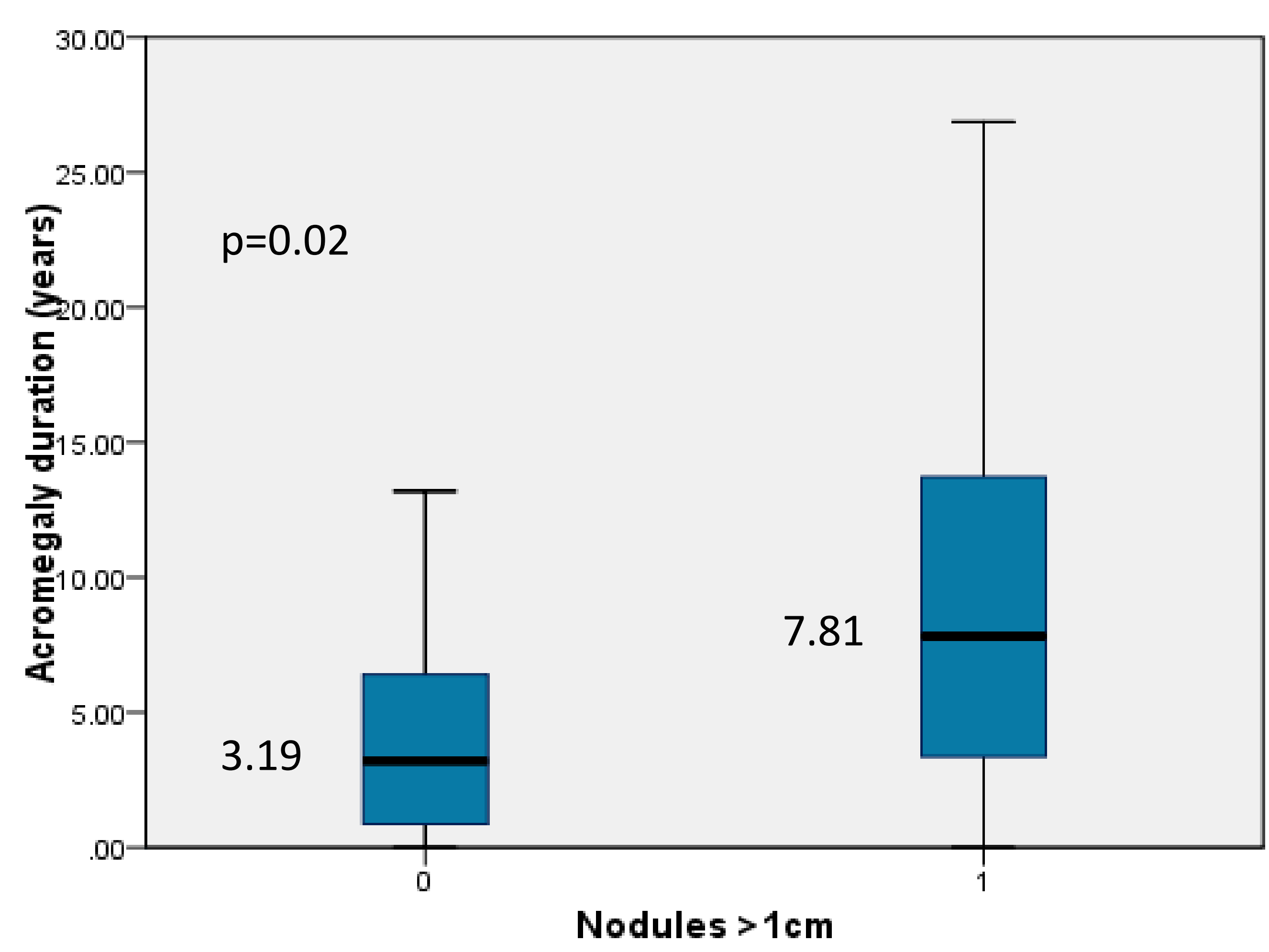

Characteristics of acromegalic patients with thyroid carcinoma

\begin{tabular}{|lllll|}
\hline Patient & Gender & Age(years) & Acromegaly duration (years) & Pathology \\
\hline 1 & $\mathrm{~F}$ & 37 & 7 & follicular variant of papillary macrocarcinoma \\
2 & $\mathrm{~F}$ & 52 & 24 & follicular variant of papillary microcarcininoma \\
3 & $\mathrm{~F}$ & 46 & 4 & follicular variant of papillary microcarcinoma \\
\hline
\end{tabular}

\section{CONCLUSION}

Due to increased prevalence of differentiated thyroid carcinoma, active screening for thyroid abnormalities is mandatory both in iodine sufficient and deficient areas, especially in patients with longer duration of acromegaly. 